\title{
VentX expression in tumor-associated macrophages promotes phagocytosis and immunity against pancreatic cancers
}

\author{
Yi Le, ${ }^{1}$ Hong Gao, ${ }^{2}$ William Richards, ${ }^{3}$ Lei Zhao, ${ }^{4}$ Ronald Bleday, ${ }^{3}$ Thomas Clancy, ${ }^{3}$ \\ and Zhenglun Zhu' ${ }^{1}$ \\ 'Department of Medicine, Brigham and Women's Hospital, Boston, Massachusetts, USA. Department of Medicine, \\ Tufts Medical Center, Boston, Massachusetts, USA. ${ }^{3}$ Department of Surgery and ${ }^{4}$ Department of Pathology, Brigham and \\ Women's Hospital, Boston, Massachusetts, USA.
}

\begin{abstract}
Pancreatic ductal adenocarcinoma (PDA) is a lethal malignancy that has no effective treatment. The tumor microenvironment (TME) of PDA employs a multitude of immune derangement strategies to protect PDA from immune elimination. Tumor-associated macrophages (TAMs) have been implicated in the pathogenesis of immune suppression of the PDA TME; however, its underlying mechanisms remained largely unknown. Using primary patient samples, our studies showed that, in comparison with macrophages isolated from normal pancreatic tissues, the phagocytosis activity of the PDA TAMs was significantly reduced. We found that the expression of homeobox protein VentX, a master regulator of macrophage plasticity, was significantly decreased in the PDA TAMs. We demonstrated that VentX was required for phagocytosis and that restoration of VentX expression in PDA TAMs promoted phagocytosis through the regulation of the signaling cascades involved in the process. Using an ex vivo culture model of primary human PDA, we showed that VentX-modulated TAMs transformed the PDA TME from a protumor milieu to an antitumor microenvironment by rectifying differentiation, proliferation, and activation of PDA-infiltrating immune cells. Using NSG-PDX models of primary human PDAs, we showed that VentX-modulated TAMs exerted strong inhibition on PDA tumorigenesis in vivo. Taken together, our data revealed a central mechanism underlying immune evasion of PDA and a potential novel venue to improve PDA prognosis.
\end{abstract}

Conflict of interest: The authors have declared that no conflict of interest exists.

Copyright: (c) 2020, American Society for Clinical Investigation.

Submitted: February 6, 2020

Accepted: June 4, 2020

Published: July 23, 2020.

Reference information: /CI Insight. 2020;5(14):e137088.

https://doi.org/10.1172/jici.

insight.137088.

\section{Introduction}

Pancreatic ductal adenocarcinoma (PDA) is one of the most lethal malignancies, with a median survival of less than 1 year and an overall 5-year survival of less than $5 \%(1,2)$. Despite exciting progress, current immunotherapeutic modalities have shown limited efficacy against PDA (3). Without an effective therapeutic option in sight, PDA mortality currently closely parallels its rising incidence and is expected to be the second leading cause of cancer-related death in $2030(4,5)$.

Over the past decades, extensive investigations indicated the role of the PDA tumor microenvironment (TME) in rendering PDA resistance to current treatment modalities (6). In contrast to that of immune-responsive cancer types, such as non-small cell lung cancer, renal cell carcinoma, and melanoma, the PDA TME demonstrated unique features, with a multitude of immune derangement to protect PDA from immune elimination $(7,8)$. In the PDA TME, there is a paucity of cytotoxic CD8 $\mathrm{T}$ cells but increased prevalence of immune-suppressive Tregs $(9,10)$. Moreover, the PDA TME contains significant numbers of tumor-associated macrophages (TAMs), which display a protumor M2-like phenotype $(11,12)$. In comparison with melanoma, PDA TAMs expresses higher levels of the immune checkpoint inhibitor, Vista, but lower levels of PD-L1 (13). Collectively, the molecular and cellular compositions of the PDA TME constitute an immune-privileged environment to protect PDA as a "cold tumor" (3). Despite speculation, the rudimentary cause of PDA immune evasion has remained largely unknown, and methods to reverse the immune suppression at the PDA TME remained out of reach.

TAMs are a major component of the PDA TME. Using clinical samples obtained from patients with primary PDA, we observed that phagocytosis, the essential macrophage function in antigen presentation 
and immune surveillance, is significantly impaired in PDA TAMs. To define the mechanism underling the TAM inertia, we found that the expression of homeobox protein VentX, a master regulator of macrophage plasticity (14), is drastically reduced in TAMs of all cases of PDA tested in this study. We show that restoration of VentX expression in PDA TAMs recovered its phagocytosis function. Mechanistically, we show that VentX promoted the expression of cell surface receptors, such as TLRs, and controls intracellular signaling cascades involved in phagocytosis. Consistent with its role in polarizing TAMs from the protumor M2-like phenotype to the antitumor M1-like phenotype, we demonstrated that VentX-modulated TAMs transform the PDA TME from immune suppressive to immune activating by alternating the molecular and cellular composition at the PDA TME. Using a preclinical model of primary human PDA, we show that VentX-modulated TAMs exerted strong inhibition of PDA tumorigenesis in vivo. Taken together, our studies suggests a key regulatory role of VentX in governing the fundamental function of macrophages and a central function of VentX-TAMs in modulating immunity at the PDA TME. Our study suggests a potentially novel venue for PDA immunotherapy, functioning in part by promoting phagocytosis and rectifying immune derangement at the PDA TME.

\section{Results}

Characterization of the PDA TME. To better understand the mechanism underlying immune suppression at the PDA TME, we characterized the composition and function of immune cells at the PDA TME. TAMs and $\mathrm{T}$ cells were isolated from fresh PDA and adjacent normal tissues, which were verified by a board-certified pathologist. Consistent with prior immunohistochemistry findings (15), in comparison with adjacent normal tissues, the PDA TME contained significantly increased numbers of macrophages, which predominately displayed a characteristic M2-like phenotype (Figure 1 and Supplemental Figure 1). Consistent with their immune-suppressive function in the PDA TME, the PDA TAMs expressed increased levels of immune-suppressive cytokines, such as IL-4, IL-10, IL-13, and TGF- $\beta$, but decreased levels of proinflammatory cytokines, IL-1 $\beta$, IL-8, IL-12B, and TNF- $\alpha$ (Figure 2A). In addition, the PDA TAMs expressed increased levels of immune checkpoint inhibitors, such as PD-L1 and Vista (Figure 2B). Similar to the TME of colorectal cancer (CRC), the prominent presence of M2-like TAMs in the PDA TME was accompanied by a significantly increased number of immune-suppressive Tregs. In contrast, there were very limited numbers of cytotoxic effector CD8 T cells in the PDA TME (Supplemental Figure 2; supplemental material available online with this article; https://doi.org/10.1172/jci.insight.137088DS1).

Vent $X$ expression is decreased in PDA TAMs. As the key executor of both innate and adaptive immunity, TAMs have been implicated in immune suppression at the TME (16). Based on our recent findings on homeobox protein VentX, a master regulator of macrophage plasticity, and its implications in the pathogenesis of immune suppression at the $\operatorname{TME}(14,17,18)$, we further explored the potential involvement of VentX in the immunopathogenesis of PDA. We found that, similar to its involvement in CRC (18), the expression of VentX is significantly decreased in TAMs of all cases of PDA tested in this study (Figure 2, C and D).

Restoration of VentX expression in PDA TAMs promotes phagocytosis. Phagocytosis constitutes the essential effector function of macrophages (19). While implicated in antitumor immunity, the role and mechanisms of phagocytosis in the pathogenesis of PDA has remained poorly defined. Using primary clinical samples, we sought to determine whether the phagocytosis function of TAMs was compromised in the PDA TME. To achieve our goal, PDA TAMs and control macrophages were isolated from PDA and adjacent normal pancreatic tissues. Using an ex vivo phagocytosis assay of CFSE-labeled pancreatic cancer cells, we found that the phagocytic activities of PDA TAMs were significantly impaired in comparison with that of the macrophages isolated from adjacent normal pancreatic tissues (Figure 3A). Consistent with its functional plasticity, previous studies suggested that phagocytosis is regulated by M1 and M2 signals (20). As such, our findings that VentX was significantly downregulated in PDA TAMs prompted us to explore whether VentX regulates phagocytic function of PDA TAMs. Therefore, we transfected the primary PDA TAMs with plasmid encoding GFP-labeled VentX (GFP-VentX, a cheremic protein of GFP and VentX) or control GFP and found that ectopic expression of VentX led to 2.5- to 3-fold increases of PDA TAM phagocytosis (Figure 3B). The effects of VentX on PDA TAM phagocytosis can be readily visualized with phase-contrast and fluorescent microscopy examination of the coculture of PDA TAMs and CellTrance Yellow-labeled tumor cells (Figure 3, B and C). To rule out the possibility that VentX promotion of phagocytosis is cell-type specific, we tested the effects of VentX expression on PDA TAM phagocytosis of leukemia cells and found that VentX promoted phagocytosis indiscriminately (Supplemental Figure 3). In addition to its effects in promoting phagocytosis, when 
A

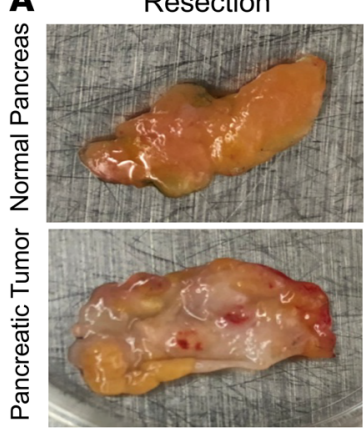

B

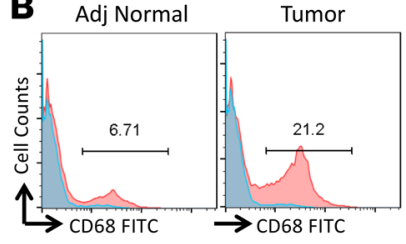

D

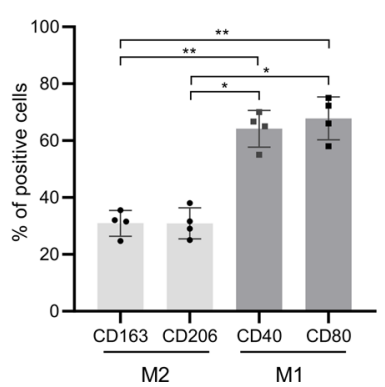

H\&E

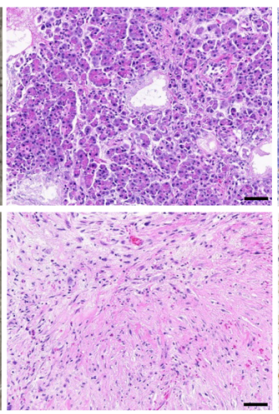

Vimentin

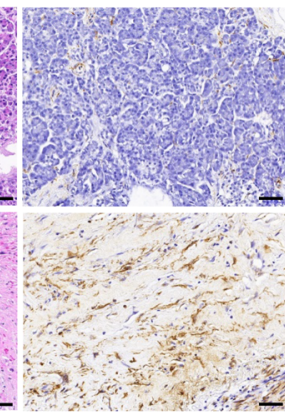

C

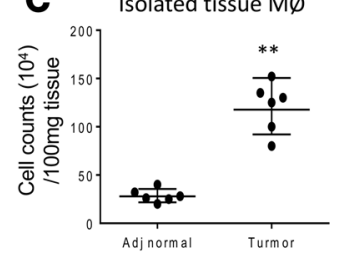

Figure 1. Characterization of pancreatic ductal adenocarcinoma.

(A) Representative images of H\&E and vimentin immunostaining of pancreatic ductal adenocarcinoma (PDA) and control adjacent normal pancreatic tissue. Scale bar: $200 \mu \mathrm{m}$. (B) FACS analysis of CD68+ macrophages in pancreatic cancers. $\mathrm{CD68} 8^{+}$macrophages in adjacent normal pancreatic tissue and PDA were analyzed by a flow cytometer. Blue shading represents isotype control, and red shading represents $\mathrm{CD}^{+} 8^{+}$ cells. (C) Accounting of $\mathrm{CD68}^{+}$macrophages in pancreatic cancer and adjacent normal pancreatic tissues. $n=6$. ${ }^{*} P<0.01$, paired Student's $t$ test. (D) The percentage of surface expression of M2 markers (CD163 and CD206) and M1 surface markers (CD40 and CD80) on CD68 ${ }^{+}$macrophages of adjacent normal pancreatic tissues and PDA TAMs from the same patients. Data are shown as the mean \pm SD of 4 independent experiments. ${ }^{*} P<0.05,{ }^{*} P<0.01,1$-way ANOVA with multiple comparisons was performed.

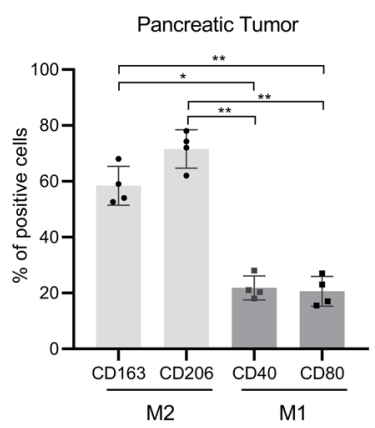

a time-course observation of phagocytosis was performed, we found that, after the CFSE-labeled cells were engulfed during the first 24 hours, the CFSE dyes became diffusely distributed throughout the cell in 24-48 hours (Figure 3C). There was no formation of foamy cells or accumulation of vacuole of uncleared phagocytic material, as observed in M2 macrophages (21). Indeed, consistent with our prior findings in CRC-TAMs (18), we found that ectopic expression of VentX in PDA TAMs promotes the polarization of PDA TAMs from the M2-like phenotype to the M1-like phenotype (Supplemental Figure 4). We showed that VentX inhibited the cell surface expression of M2 marker CD163 but promoted the expression of M1 marker CD80 (Supplemental Figure 4A); VentX also promoted the expression and secretion of proinflammatory cytokines TNF- $\alpha$, IL-1 $\beta$, and IL-12 but inhibited the expression of immune-suppressive cytokines TGF- $\beta$, IL- 4 , and IL-10 (Supplemental Figure 4, C and D). Moreover, we found that VentX expression in PDA TAMs inhibited the expression of immune checkpoint blockade proteins PD-L1 and Vista and promoted the expression and secretion of effector molecules, such as nitrate (Supplemental Figure 4, B and E).

To explore the mechanisms underlying VentX promotion of phagocytosis, we performed a targeted screen of cell surface receptors and signaling pathways implicated in phagocytosis. We found that VentX promoted the expression of TLR2 and TLR9 and downstream signaling molecules MyD88 and MAP kinase p38 in PDA TAMs (Figure 4A). In comparison, ectopic expression of VentX in PDA TAMs exerted little effect on the expression of SIRP $\alpha$, the cellular receptor for CD47, the "do-not-eat-me" signal implicated in cancer immune evasion (22) (Figure 4B). Instead, we found that VentX expression inhibits the phosphorylation of SHP-1 and SHP-2, the SIRP $\alpha$ downstream signaling molecules implicated in inhibition of phagocytosis. Moreover, we found that VentX expression promoted the phosphorylation of FAK kinase implicated in promoting phagocytosis (Figure 4C). Consistent with the essential requirement of VentX in the phagocytic function of PDA TAMs, we found that when the PDA TAMs were treated with morpholino-oligo against VentX, the stimulation effects of LPS were abolished (Figure 4D). Our findings suggested an essential role of VentX in regulating the balance of signaling cascades to promote phagocytosis. 

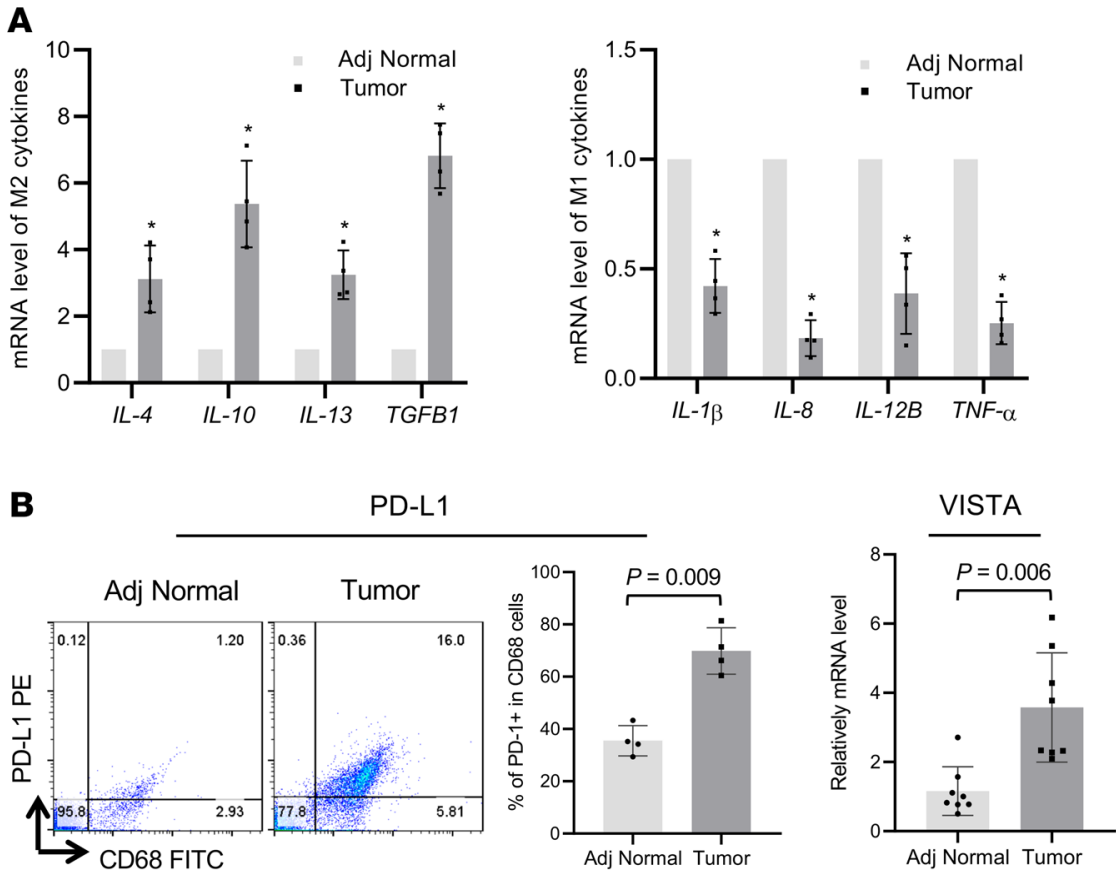

$$
\text { C }
$$
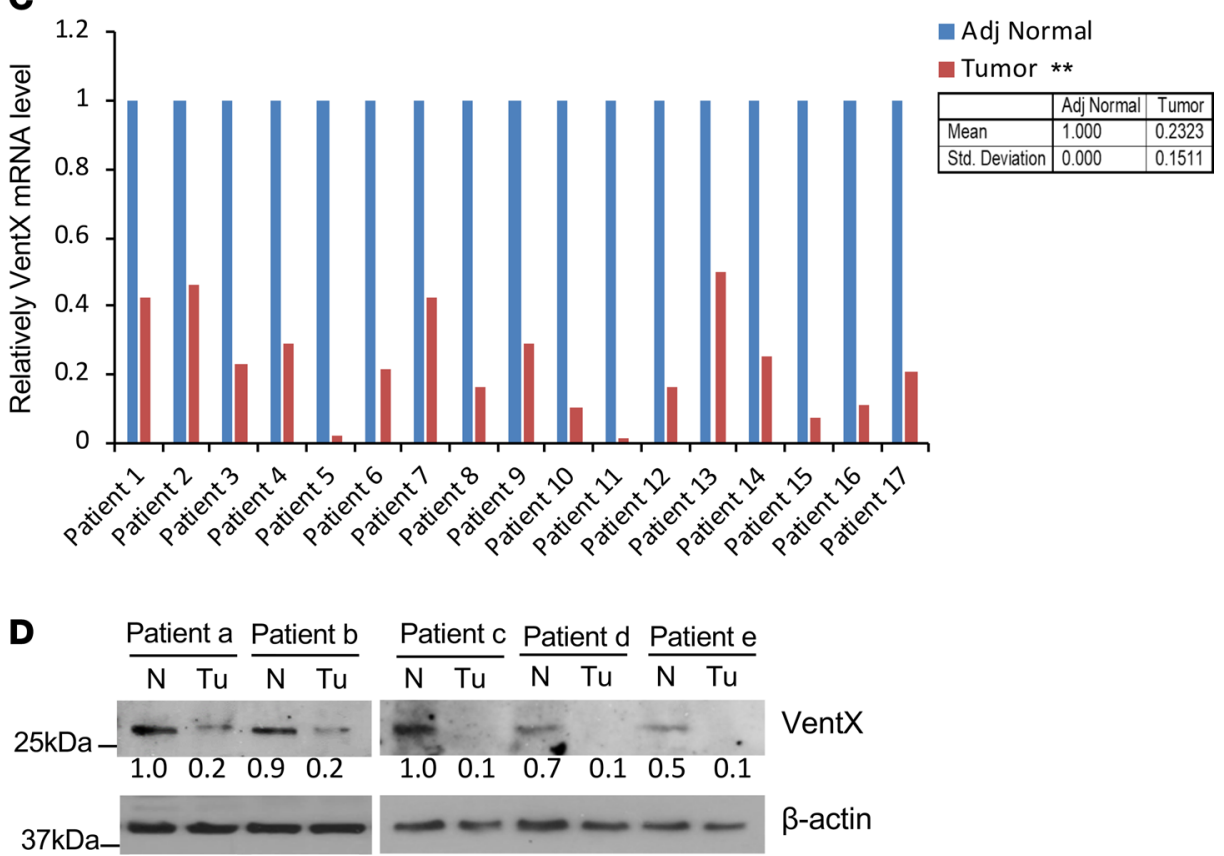

Figure 2. Characterization of tumor-associated macrophages in pancreatic ductal adenocarcinoma. (A) The mRNA expression levels of indicated M1 and M2 cytokines in tumor-associated macrophages (TAMs) versus control macrophages, as determined by qRT-PCR. Results represent mean \pm SD of 4 independent experiments. ${ }^{*} P<0.05$, paired Student's $t$ test. (B) Cell surface expression of checkpoint inhibitor PD-L1 and Vista mRNA in TAMs and control macrophages isolated from adjacent normal pancreatic tissues. The expression level of PD-L1 was determined by flow cytometer analysis, and representative graphs are shown. $n=4$. The mRNA expression levels of Vista were determined by qRT-PCR. Results represent mean \pm SD of 8 independent experiments. $P<0.01$, paired Student's $t$ test. (C) Paired comparison of qRT-PCR measurements of VentX mRNA expression in macrophages isolated from normal control tissues and TAMs of 17 patients. The relative VentX mRNA expression levels in normal macrophages were arbitrarily designated as $1 .{ }^{* *} P<0.01$, TAMs vs. adjacent normal macrophages, paired Student's $t$ test. $n=17$. (D) Western blot analysis of endogenous VentX protein levels in macrophages isolated from normal control tissues and TAMs of 3 patients. The numbers indicate the relative fold difference of VentX proteins, as determined by ImageJ (NIH) software scanning. N, normal control tissue; Tu, tumor. 
A
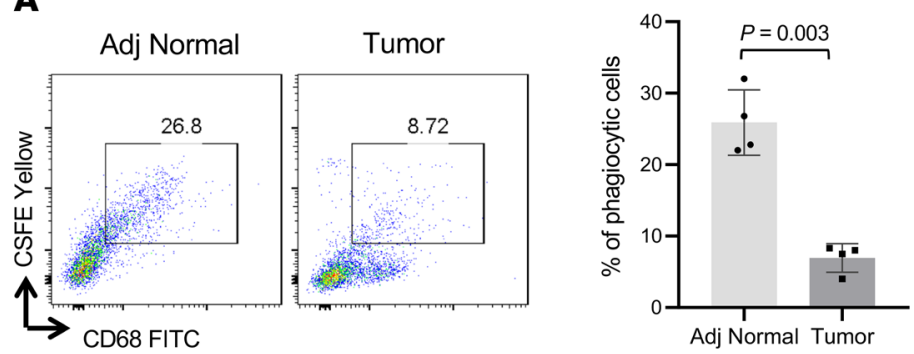

B GFP TAM

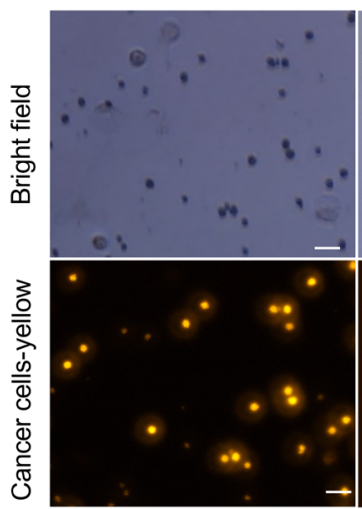

GFP-VentX TAM
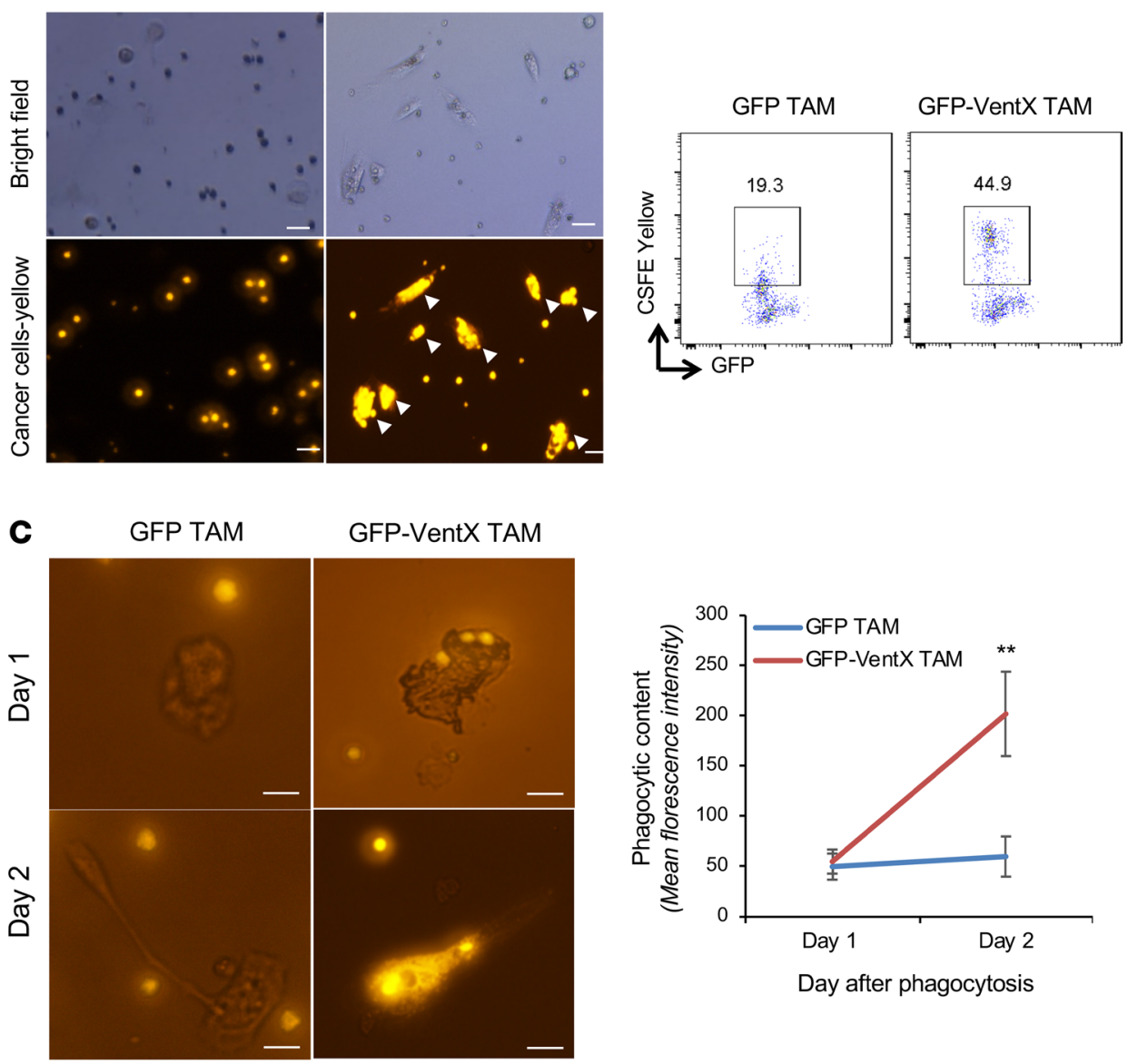

Figure 3. VentX promotes TAM phagocytosis. (A) Quantification of phagocytosis activity of TAMs and control macrophages. Freshly isolated TAMs and control macrophages were incubated with $1 \mu \mathrm{M}$ CellTrance Yellow-labeled Panc-1 cells. The rate of phagocytosis was determined by flow cytometry. Data are shown as the mean $\pm \mathrm{SD}$ from 4 independent experiments. $P<0.01$, paired Student's $t$ test. (B) VentX promotes TAM phagocytosis. TAMs were isolated and transfected with plasmids encoding GFP or GFP-VentX. The transfected TAMs were then incubated with $1 \mu \mathrm{M}$ CellTrance Yellow-labeled cancer cells for 24 hours. The effects of the treatment were determined by phase-contrast and fluorescent microscopy (left) and flow cytometry analysis (right). Arrowheads indicated phagocytosed cancer cells. Scale Bar: $50 \mu \mathrm{M}$. The representative figure was shown. $n=3$. (C) Phagocytosis and intracellular digestion of CellTrance Yellowlabeled Panc- 1 cells. TAMs transfected with control GFP or GFP-VentX were incubated with CellTrance Yellow-labeled Panc- 1 cells. Representative images of the phagocytosis were revealed by fluorescent microscopy after 24 hours (top) and 48 hours (bottom). Scale Bar: $20 \mu \mathrm{m}$. Quantification of phagocytotic cells was performed by measuring green fluorescence intensity after phagocytosis. The graph represents the pixel intensity of fluorescence. Data presented are mean $\pm \mathrm{SD} . n=3 .{ }^{* *} P<0.01$. At least 10 cells were scanned for each time point.

VentX-TAMs transforms the PDA TME and promotes immunity against PDA. A multitude of immune derangements of the PDA TME has been implicated in PDA evasion of current immunotherapeutic modalities. Macrophages are key executor of both innate and adaptive immunity. Our findings that VentX promoted PDA TAM phagocytosis and polarized PDA TAMs from the M2-like phenotypes to the M1-like phenotypes prompted us to examine the effects of VentX-modulated PDA TAMs on the immune state of the PDA TME. Fresh isolated PDA TAMs were transfected with plasmid encoding GFP-VentX (VentX-TAMs) or control GFP (Control-TAMs). The VentX-TAMs and the control-TAMs were then cocultured with en block autologous PDA tissues. The composition of immune cells at the PDA TME was analyzed 5 days later. We found that in comparison with the control-TAMs, coculturing VentX-TAMs led to an approximately 3-fold increase of the M1-like TAMs and a more than 5-fold decrease of the M2-like TAMs in the PDA TME (Figure 5A). In parallel to the switch of the M1 versus M2 ratio, there was about a 4-fold reduction in the number of inhibitory Tregs and more than a 4-fold increase of the cytotoxic CD8 T cells in the PDA TME (Figure 5, B and C). Besides FACS analysis, we also examined the immune cell composition of the PDA TME by immunohistochemistry. Consistent with the FACS findings, immunohistochemistry analysis of a thin section of PDA before and after the coculturing with VentX-TAMs showed a dramatic reduction 
A

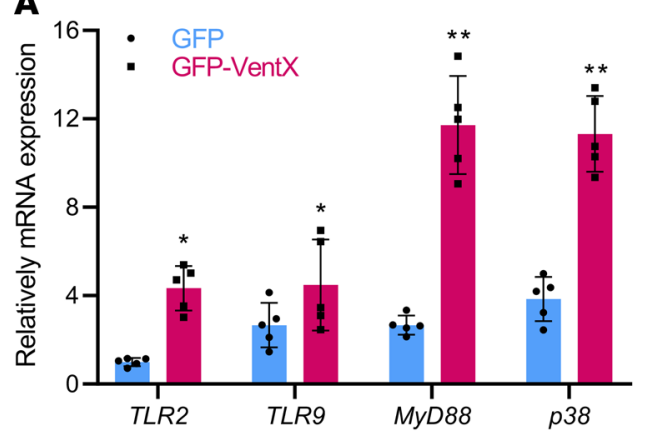

B

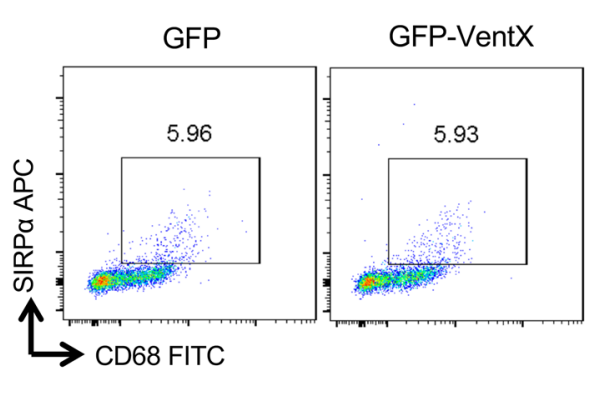

C

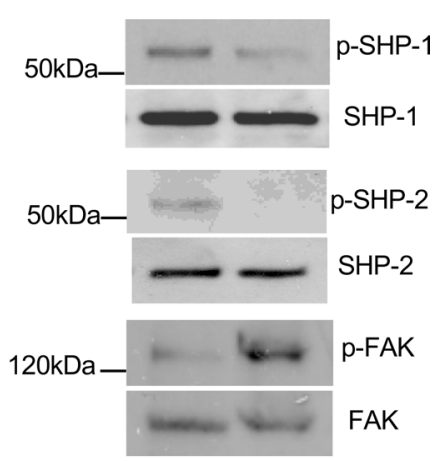

D Control LPS

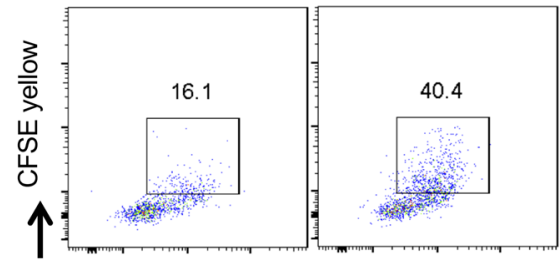

Control-MO + LPS VentX-MO + LPS
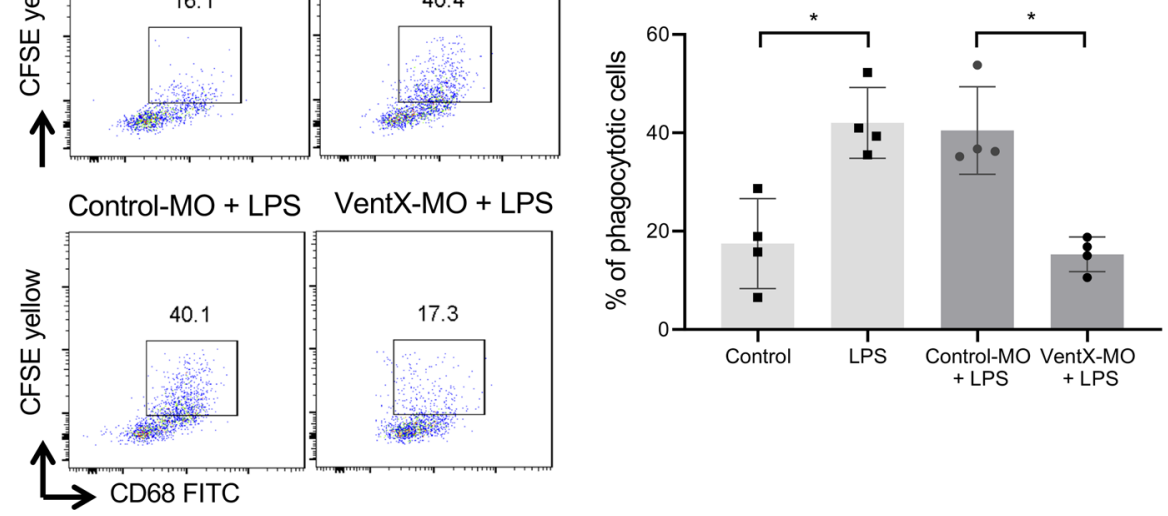

Figure 4. Effects of VentX on the expression of cell surface receptors and signaling molecules implicated in phagocytosis. (A) Effects of VentX on the expression of TLRs and signaling molecules. Pancreatic TAMs were transfected with plasmids encoding GFP control and GFP-VentX. The expression of indicated TLRs and signaling molecules was determined by qRT-PCR. Data are shown as the mean \pm SD of 5 different experiments. ${ }^{*} P<0.05$, ${ }^{* *} P<0.01$, paired Student's $t$ test. (B) FACS analysis of SIRP $\alpha$ receptor expression on TAMs transfected with plasmids encoding GFP or GFP-VentX. (C) Western blot analysis of phosphorylation state as well as total protein of the intracellular signaling molecules SHP-1, SHP-2, and FAK in GFP- and GFP-VentX-transfected TAMs. (D) Requirement of VentX for LPS stimulation of TAM phagocytosis. TAMs were isolated and transfected with morpholino oligo against VentX or control morpholino (MO) and then subjected to LPS stimulation. The effects of the treatment on phagocytosis were determined by flow cytometry.

of the M2-like TAMs and marked increases in the number of cytotoxic CD8 T cells (Figure 5D). To explore the mechanisms underlying the alternation of cellular composition at the PDA TME, we cocultured CD8 $\mathrm{T}$ cells with autologous PDA TAMs transfected with GFP-VentX or control GFP and found that VentX abolished the inhibitory effects of PDA TAMs on CD8 proliferation and activation (Supplemental Figure 5). Together with the effects of VentX on PDA TAM phenotypes and the expression of immune checkpoint inhibitors (Supplemental Figure 4), our data suggested that VentX-TAMs transform the immune status of the PDA TME by rectifying a multitude of molecular and cellular derangements at the PDA TME. As the $\mathrm{M} 1 / \mathrm{M} 2$ ratio and the $\mathrm{CD} 8$ /Treg ratio have been implicated in PDA prognosis (11), we sought to explore the therapeutic potential of VentX-TAMs on PDA. As VentX does not have a murine homolog (23), we transplanted a small piece of primary human PDA tissue subcutaneously at the dorsal lateral side of NSG mice to generate the NSG patient-derived xenograft (NSG-PDX) model of primary human PDA. Seven days after transplantation, the NSG-PDX model of primary human PDA (NSG-PDA) mice were tail vein injected with VentX-TAMs or control-TAMs. Consistent with the tumor chemokine-traction of macrophages (24, 25), we found that the injected TAMs accumulated in lymph nodes on the side of transplanted tumors (Figure 6A). In addition, we also observed the homing of the injected VentX-TAMs to the implanted PDA (Supplemental Figure 6B). The effects of VentX-TAMs on the growth of PDA in vivo was observed for up to 6 weeks. As shown in Figure 6B, in comparison with the mice treated with control-TAMs, VentX-TAMs exerted strong inhibition on the growth of PDA in the NSG-PDX model of primary human PDA. 
A

GFP

GFP-VentX

Gated on CD68+ cells
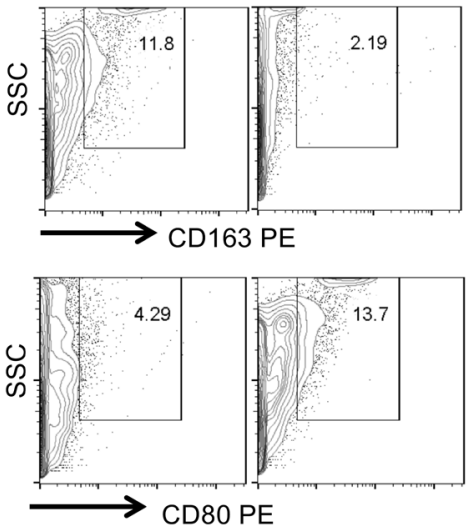

C

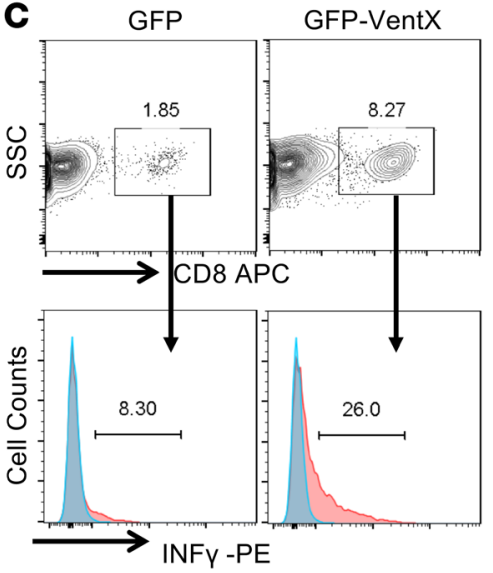

B GFP GFP-VentX Gated on CD4+ T
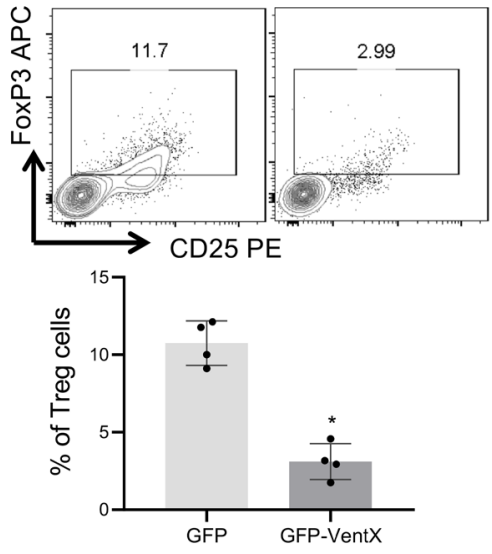

D

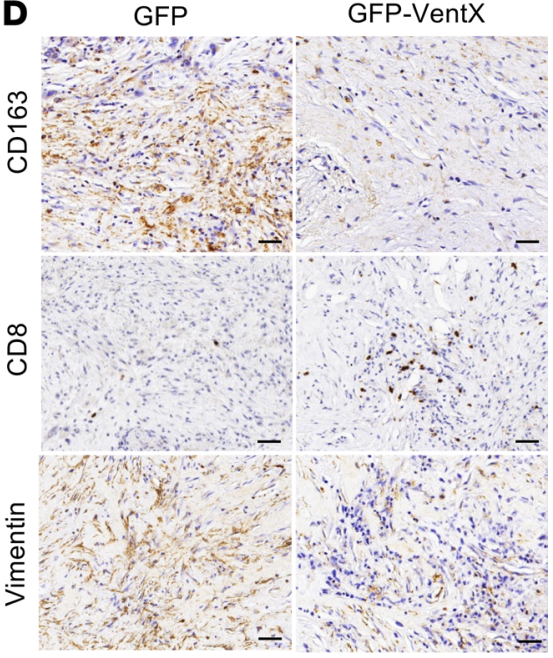

Figure 5. Effects of VentX-TAMs on PDA infiltrating immune cells at the TME. (A) VentX-TAMs promotes M2 to M1 transition of PDA TAMs. En block PDA tumor tissues were incubated with autologous TAMs transfected with GFPVentX or GFP control. After 5-day incubation, the tumor endogenous TAMs were isolated, and the percentage of M1and M2-like TAMs was determined by FACS analysis of respective cell surface markers, CD80 and CD163. (B) The effect of the incubation on Treg differentiation was determined by FACS analysis of percentage of $C D 4^{+} C D 25^{+}$Foxp3 $3^{+}$cells. Data represent the mean \pm SD from 4 independent experiments. ${ }^{*} P<0.05$, paired Student's $t$ test. (C) The effect of the incubation on proliferation (top) and activation (bottom) of CD8 $8^{+}$TIL cells was determined by FACS analysis of CD8 marker and IFN- $\gamma$ expression, respectively. (D) Effects of VentX-TAM incubation on differentiation of PDA and infiltrating immune cells, as revealed by immunohistochemical staining. Representative images are shown. Anti-CD163, -CD8, and -vimentin antibodies were used for the immune staining, as indicated. Scale bar: $100 \mu \mathrm{m}$.

\section{Discussion}

Phagocytosis underlies the fundamental function of macrophages for antigen presentation and immunosurveillance. However, the role of phagocytosis in tumorigenesis remains an area of active debate (26). Previous studies suggested that inhibition of phagocytosis may play an important role in tumor evasion of immune surveillance. It was shown that the expression of CD47, the do-not-eat-me signal, is elevated in a variety of human cancers. On the other hand, the expression of CD163, a scavenger receptor, is increased in the M2-like TAM, and studies suggested that M2 macrophages phagocytose antibody-opsonized target cancer cells more efficiently than M1 macrophages $(27,28)$. Despite speculation, systemic examination of macrophage function in the pathogenesis of PDA has been scarce. In our current studies, using primary human pancreatic cancer as a model, we found that the phagocytosis function of PDA TAMs is significantly impaired in comparison with macrophages isolated from control normal pancreatic tissue. We found that VentX expression is drastically 
A
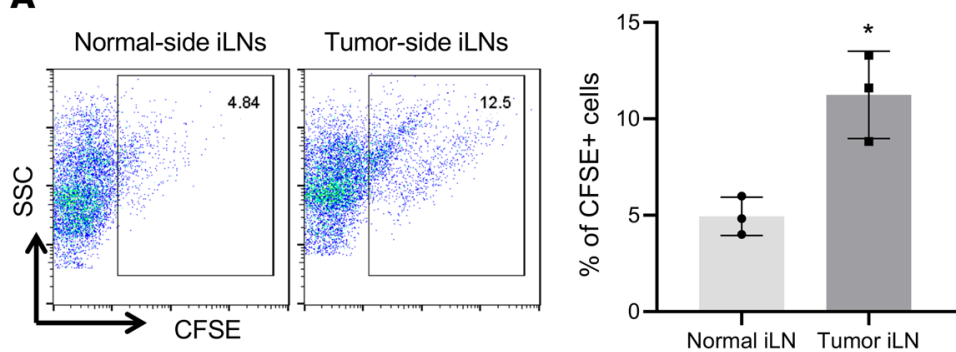

B

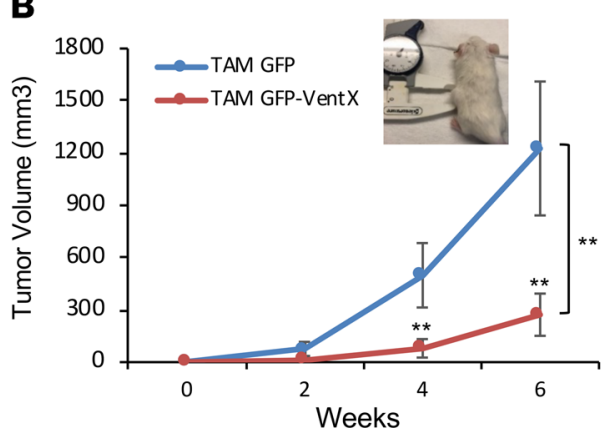

Figure 6. VentX-TAMs inhibits tumorigenesis of PDA in NSG-PDX models of primary human pancreatic cancers. NSG-PDX models of primary human pancreatic cancers were generated by subcutaneous implantation of small pieces of primary human PDA on the dorsal side of NSG mice. The mice were then tail vein injected with VentX-TAMs or control GPF-TAMs. (A) Distribution of VentXTAMs in lymph nodes. The CFSE-labeled VentX-transfected TAMs were tail vein injected into NSG-PDX mice with human PDA. 72 hours after injection, inguinal lymph nodes (iLN) from the tumor side or control side were removed from the mice, and single-cell suspensions were analyzed by flow cytometry. The percentage of CFSE-positive TAMs is shown. Data are shown as the mean \pm SD of 3 independent experiments. ${ }^{*} P<0.05$, paired Student's $t$ test. (B) The growth of the PDA in vivo was observed for up to 6 weeks, and the results of the treatment are shown. Data represent the mean of 7 independent experiments $(n=7)$. ${ }^{* *} P<0.01$, 2-way ANOVA with repeated measures.

reduced in TAMs of all cases of PDA tested in this study. The importance of these studies is suggested by our the findings that restoration of VentX expression in PDA TAMs revokes the phagocytosis function of TAMs and that VentX-TAMs transform immunity by alternating the molecular and cellular composition at the PDA TME. Interestingly, mechanistic exploration showed that VentX expression did not alter the expression of SIRP, the cellular receptor of CD47. On the other hand, we did find that ectopic expression of VentX inhibits phosphorylation of SHP-1 and SHP-2, SIRP downstream signaling molecules implicated in the inhibition of phagocytosis. Moreover, our data showed that VentX promotes the expression of TLRs and downstream signaling molecules as well as FAK signaling, which has been implicated in promoting phagocytosis. Our data are consistent with the prior notion of M1 and M2 signaling in the regulation of phagocytosis. We show here that this process is controlled by VentX, a human homolog of the Xenopus ventral homeobox protein Xom (29-31). Since the initial discovery of macrophages as the key regulator of immunity more than one hundred years ago, the cell-intrinsic factor that controls macrophage plasticity has remained elusive $(32,33)$. By leveraging the power of developmental study, which led to the discovery of the VentX as the key regulator of macrophage plasticity $(14,17,18,30,31,34)$, our current findings suggested a fundamental mechanism of immune regulation, which underlies the central mechanism of immune suppression at the PDA TME. Currently, the mechanism underlying decreased VentX expression in PDA TAMs remains unknown. Our data suggest that further exploration along these lines may provide better mechanistic insight and potentially novel therapeutic opportunities to improve PDA prognosis.

\section{Methods}

Collection of pancreatic tissue samples. A total of 19 patients with pancreatic adenocarcinoma, who were scheduled for surgical resection at Brigham and Women's Hospital, consented to have a portion of resected tissues and blood collected for research purposes. The characteristics of the patients with pancreatic cancer, whose specimens were used for this study, are listed in Table 1 . Approximately 5-10 g tissues were collected from the tumor mass or adjacent normal tissues. Tumor samples and control tissues were verified by board-certified pathologists at Brigham and Women's Hospital.

Preparation of lymphocytes and macrophages from pancreatic and tumor tissues. Lymphocytes were isolated essentially as described previously $(14,18,35)$. Briefly, dissected fresh normal pancreatic tissues and pancreatic tumors were rinsed in a $10-\mathrm{cm}$ Petri dish with $\mathrm{Ca}^{2+}$-free and $\mathrm{Mg}^{2+}$-free $\mathrm{HBSS}$ (Life Technologies) containing 2\% FBS and $2 \mathrm{mM}$ Dithiothreitol (MilliporeSigma). The pancreatic and tumor tissues were then cut into approximately $0.1-\mathrm{cm}$ pieces with a razor blade and incubated in $5 \mathrm{~mL}$ HBSS containing $5 \mathrm{mM}$ EDTA (MilliporeSigma) at $37^{\circ} \mathrm{C}$ for 1 hour. The tissues were then passed through a gray mesh (100 microns). The flow-throughs containing lymphocytes and epithelial cells were then analysis by a flow cytometer. 
To isolate the macrophages, normal pancreatic tissues and pancreatic tumors were rinsed with HBSS, cut into approximately $0.1-\mathrm{cm}$ pieces with a razor blade, and then incubated in HBSS (with $\mathrm{Ca}^{2+} \mathrm{and}^{\mathrm{Mg}} \mathrm{Mg}^{2+}$ ) containing 2\% FBS, $1.5 \mathrm{mg} / \mathrm{mL}$ Collagenase D (Roche), and $0.1 \mathrm{mg} / \mathrm{mL}$ Dnase I at $37^{\circ} \mathrm{C}$ for 1 hour. The digested tissues were then passed through a gray mesh (70-micron) filter. The flow-throughs were collected, washed, and resuspended in RPMI 1640 medium. Normal tissue macrophages and TAMs were further purified using the EasySep Human Monocyte/Macrophage Enrichment kit without CD16 depletion (StemCell Technologies, catalog 19085) according to the manufacturer's instructions. The isolation process did not lead to activation of macrophages, and the purity of isolated macrophages was above $95 \%$ (14, 35, 36). Greater than $98 \%$ of cells isolated were viable by propidium iodide staining tests.

FACS analysis. Phenotypic analysis of macrophages and lymphocytes was performed using flow cytometry after immunolabeling of cells with fluorescence dye-conjugated antibodies. The following antibodies were used: PE-conjugated anti-CD3 (OKT3), -CD25 (BC96), -CD14 (61D3), -CD40 (5C3), -CD80 (2D10.4), -CD163 (GH161), and -PD-1L (J105); FITC-conjugated anti-CD4 (RPA-T4) and -CD68 (Y182A); and APC-conjugated anti-CD8 (OKT8), -CD4 (OKT4), and -SIRP $\alpha$ (clone 15-414) (eBioscience). Intracellular staining of anti-Foxp3 (236A/E7) APC and -IFN- $\gamma$ (4S.B3) PE antibodies was performed following the protocol provided by the manufacturer (eBioscience). Isotope control labeling was performed in parallel. Antibodies were diluted as recommended by the manufacturer (eBioscience). Labeled cells were acquired using the BD LSRFortessa at the Flow Cytometry Core of the Dana-Farber Cancer Institute with FACS Diva software (BD Biosciences) and analyzed using FlowJo 10.1 software (Tree Star). Typically, 20,000 cells were analyzed per sample according to the standard FACS analysis procedure. Results are expressed as the percentage of positive cells.

Quantitative RT-PCR. Total RNA was isolated by the TRIzol reagent (Life Technologies), and RNA amounts were measured by NanoDrop 2000 (Thermo Scientific). Equal amounts of RNA were used for first-strand cDNA synthesis with the SuperScript III First-Strand Synthesis System (Life Technologies) according to the manufacturer's protocol. The AccuPrime Taq DNA polymerase system (Life Technologies) was used to amplify VentX cDNA with conventional PCR. Quantitative measurements of VentX and other cDNA were carried out with SYBR Green (LightCycler 480 SYBR Green Master kit, Roche Life Science), using a Mastercycler ep Gradient S (Eppendorf). GAPDH was used as a housekeeping gene to normalize mRNA expression. The primers used are listed in Supplemental Table 1. Relative expression profiles of mRNAs was calculated using the comparative Ct method (DDCT method).

Cytokine measurement. Levels of IL-1 $\beta$ and TNF- $\alpha$ were quantified using ELISA kits obtained from eBioscience. Analyses were conducted according to the manufacturer's instructions. Triplicate wells were plated for each condition. Nitrite concentrations in tissue culture supernatants were determined using the Griess reagent kit (Molecular Probes), as described previously (14).

Transfection assays. Plasmids encoding GFP-VentX and control GFP were described previously (31). Transfection of GFP-VentX and GFP into macrophages was carried out using the Human Macrophage Nucleofector Kit (VVPA-1008, Lonza). Briefly, $2 \times 10^{6}$ cells were resuspended in $100 \mu 1$ nucleofector solution with $5 \mu \mathrm{g}$ plasmid DNA for 20 minutes on ice. Transfections were performed on a Nucleofector $2 \mathrm{~b}$ Device (Lonza). After transfection, cells were placed on ice immediately for 1 minute and then cultured in prewarmed RPMI 1640 complete medium, containing 10\% FBS and 1\% antibiotic-antimycotic solution (Gibco, 15240062) for 48 hours before transfected cells were used for experiments.

For VentX knockdown experiments, pancreatic TAMs were transfected with morpholino oligonucleotides (MOs) (Gene Tools) using the Human Macrophage Nucleofector Kit (Lonza) as previously described $(14,18)$. Briefly, $2 \times 10^{6}$ cells were resuspended in $100 \mu$ nucleofector solution, with $2.5 \mathrm{nmol}$ of either VentX MO (5'-TACTCAACCCTGACATAGAGGGTAA-3') or a control MO oligonucleotide (5'-CCTCTTACCTCAGTTACAATTTATA-3') and electroporated with the Nucleofector II Device (Lonza). Cells were then immediately removed from the device and placed on ice for 1 minute. The cells were then incubated overnight with $1 \mathrm{~mL}$ prewarmed Human Monocyte Nucleofector Medium (Lonza Bioscience) containing $2 \mathrm{mM}$ glutamine and 10\% FBS. Cells were then resuspended in complete RPMI medium and stimulated with $10 \mathrm{ng} / \mathrm{mL}$ LPS (MilliporeSigma) or control PBS overnight for phagocytosis assays.

Phagocytosis assays. For the phagocytosis assay, PANC-1 cells (human pancreatic cancer cell line, MilliporeSigma) or primary human leukemia cells (Brigham and Women's Hospital) were labeled with $1 \mu \mathrm{M}$ CellTrance Yellow reagent using the CellTrace CFSE proliferation kit (Thermo Fisher Scientific). $5 \times 10^{5}$ TAMs transfected with GFP or GFP-VentX were plated in each well of 12-well tissue culture plates (Cor- 
Table 1. Characteristics of patients with pancreatic cancer, whose specimens were used in this study

\begin{tabular}{|c|c|c|}
\hline Characteristics & Number $(n=19)$ & $(\%)$ \\
\hline \multicolumn{3}{|l|}{ Age (y) } \\
\hline$\leq 60$ & 2 & 10.5 \\
\hline $60-79$ & 15 & 79.0 \\
\hline$\geq 80$ & 2 & 10.5 \\
\hline \multicolumn{3}{|l|}{ Sex } \\
\hline Male & 12 & 63.2 \\
\hline Female & 7 & 36.8 \\
\hline \multicolumn{3}{|l|}{ Pathology type } \\
\hline Adenocarcinoma & 19 & 100 \\
\hline \multicolumn{3}{|l|}{ Stage } \\
\hline I & 4 & 21.1 \\
\hline II & 12 & 63.2 \\
\hline III & 3 & 15.7 \\
\hline IV & 0 & 0 \\
\hline
\end{tabular}

ing). The TAMs were then incubated in RPMI complete medium for 2 hours before mixing with $1 \times 10^{6}$ of CSFE Yellow-labeled cancer cells. After the indicated incubation time, the TAMs were washed repeatedly and detached with $0.25 \%$ Trypsin/EDTA; phagocytosis was analyzed by a flow cytometer. Microscopic analysis of phagocytosis was performed with a Nikon Eclipse Ti fluorescence microscopy. Images were captured at magnifications of $\times 20$ or $\times 40$ using a color camera and the NIS Elements imaging software (Nikon). Brightness and contrast for representative images were adjusted equally among groups.

Western blot analysis. Western blot analysis was performed as described previously $(14,18)$. Briefly, cells were lysed in 1× RIPA buffer (Abcam) mixed with phosphatase and protease inhibitor cocktails (Cell Signaling Technology [CST]). Proteins were resolved by 4\%-15\% Tris-Glycine Gel (Bio-Rad) electrophoresis. Primary antibodies used included VentX (Abcam, ab105352, 1:500), $\beta$-actin (CST, 4967, 1:2000), anti-phospho-SHP-1 (Tyr564) (CST, catalog 8849, 1:500), SHP-2 (Tyr542) (CST, catalog 3751, 1:500), and phosphor-FAK (Tyr397) (CST, catalog 3283, 1:500) antibodies. Their protein control antibodies included SHP-1 (CST, catalog 3759, 1:1000), SHP-1 (CST, catalog 3397, 1:1000), and FAK (CST, catalog 3285, 1:1000). The relative fold difference of the band density was determined by ImageJ software (NIH).

$T$ cell proliferation assays. T cell proliferation was performed essentially as described previously $(18,37)$. Briefly, blood CD8 cells of pancreatic patients were enriched by the Easysep human CD8 enrichment kit (StemCell Technologies, catalog 19053) following the manufacturer's instructions. $1 \times 10^{6}$ CD8 cells were labeled with $1 \mu \mathrm{M}$ of CellTrance Yellow and then activated with human T-Activator CD3/CD28 Dynabeads (Gibco, catalog 11131D) at a bead-to-T cell ratio of $1: 4$ in the presence of $10 \mathrm{ng} / \mathrm{mL} \mathrm{IL}-2$ (PeproTech). Activated CD8 was then mixed with $0.25 \times 10^{6}$ GFP-VentX- or GFP-transfected TAMs of the same patients. The mixtures were incubated in completed RPMI 1640 at $37^{\circ} \mathrm{C}, 5 \% \mathrm{CO}_{2}$ for 5 days. Cells were then stained with an anti-CD8-APC-conjugated antibody and analyzed by a flow cytometer.

Cocultures of tumor tissues and macrophages. Tumor tissues were washed with $1 \times$ PBS buffer plus antibiotics and then cut into 0.5 -cm pieces. Tissues were mixed and cultured with $0.5 \times 10^{6} \mathrm{GFP}$-VentX- or GFP-transfected macrophages of the same patient in $2 \mathrm{~mL}$ RPMI 1640 complete medium, supplemented with 1\% antibiotic-antimycotic solution (Gibco). The cultures were incubated at $37^{\circ} \mathrm{C}, 5 \% \mathrm{CO}_{2}$, for 5 days. The tissues were then subjected to cell isolation and analyzed by a flow cytometry or immunohistochemistry studies.

NSG-PDX model of human pancreatic cancers. Animal models of primary human pancreatic cancers were developed as described previously (38). Briefly, 8-week-old NOD.Cg-Prkdscid Il2 $\mathrm{rg}^{\text {tml } 1 \mathrm{Wl} l} / \mathrm{SzJ}$ mice (commonly known as NOD/SCID/ $\gamma[\mathrm{NSG}]$ mice) were purchased from The Jackson Laboratory and maintained under specific pathogen-free conditions. Pancreatic tumors were cut into about 0.5 -cm pieces and then surgically implanted into subcutaneous space on the dorsal side of NSG mice. After 1 week of implantation, $0.5 \times 10^{6}$ TAMs transfected with GFP-VentX or control GFP were injected into the mice through tail vein. The tumor growth was monitored twice weekly and measured by a caliper for 6 weeks.

In vivo tracing of injected TAMs was carried out using CFSE-stained TAMs or macrophages, according to the manufacturer's instructions (Molecular Probes). Briefly, $1 \times 10^{6}$ TAMs were incubated in $5 \mathrm{~mL}$ of 
$5 \mu \mathrm{M}$ CFSE staining solution in a $37^{\circ} \mathrm{C}$ water bath for 20 minutes. Cells were then incubated with $20 \mathrm{~mL}$ RPMI 1640 complete medium for 5 minutes to removed unbounded dye. After centrifugation, cells were dissolved in PBS and then tail-vain injected into NSG mice. Tumor tissues and lymph nodes were dissected out 3 days after injection, and TAMs were isolated as CFSE-positive cells.

Immunohistochemistry. Immunohistochemistry was performed following established protocols (39). Briefly, pancreatic tumors or adjacent normal tissues were fixated in formalin (Thermo Fisher Scientific) for at least 48 hours. The tissues were then embedded in paraffin and sectioned. CD8 (Dako, clone C8/144B, 1:100), CD163 (Vector, clone 10D6, 1:250), Vimentin (Dako, clone M0725, 1:50), and H\&E staining was performed at the Specialized Histopathology Core at Dana-Farber/Harvard Cancer Center. All immunohistochemistry was performed on the Leica Bond automated staining platform, using the Leica Biosystems Refine Detection Kit with EDTA antigen retrieval. The images of whole slides were scanned by a Pannoramic MIDI II digital slide scanner and analyzed with Caseviewer and Quant center software (3DHistech).

Statistics. Two-tailed Student's $t$ test or 1-way ANOVA were used for statistical analysis in Prism (version $8, \mathrm{GraphPad})$. Data are presented as mean $\pm \mathrm{SD}$. Tumor growth curves were analyzed by repeated measurement 2-way ANOVA using Holm-Šidák multiple comparison test. The level of significance was indicated by the $P$ value. $P$ values of 0.05 or less were considered statistically significant.

Study approval. All patients provided written informed consent, and the Institutional Review Board of the Brigham and Women's Hospital approved these studies (2006P1354). Tumor samples and control tissues were verified by board-certified pathologists at Brigham and Women's Hospital. All animal experiments were approved by the Institutional Animal Care and Use Committee at the Brigham and Women's Hospital (2016N000353).

\section{Author contributions}

YL, HG, and ZZ designed the research, analyzed the data, and prepared figures. YL and HG performed the experiments. RB, WR, LZ, and TC provided tumor samples, clinical information, and analysis. ZZ wrote the manuscript.

\section{Acknowledgments}

We thank Marc Kirschner for critical discussion and Paul Yu for the use of equipment. We thank the Dana-Farber/Harvard Cancer Center for the use of the Specialized Histopathology Core, which provided histology and immunohistochemistry services. The Dana-Farber/Harvard Cancer Center is supported in part by an National Cancer Institute Cancer Center Support Grant (5 P30 CA06516). ZZ was supported by research funds from Brigham and Women's Hospital.

Address correspondence to: Zhenglun Zhu, Department of Medicine, Brigham and Women's Hospital, 75 Francis Street, Boston, Massachusetts 02115, USA. Phone: 617.732.5467; Email: zzhu@partners.org.

1. Hidalgo M. Pancreatic cancer. N Engl J Med. 2010;362(17):1605-1617.

2. Polireddy K, Chen Q. Cancer of the pancreas: molecular pathways and current advancement in treatment. J Cancer. 2016;7(11):1497-1514

3. Soares KC, et al. PD-1/PD-L1 blockade together with vaccine therapy facilitates effector T-cell infiltration into pancreatic tumors. J Immunother. 2015;38(1):1-11.

4. Rawla P, Sunkara T, Gaduputi V. Epidemiology of pancreatic cancer: global trends, etiology and risk factors. World J Oncol. 2019;10(1):10-27.

5. Chen W, et al. Cancer statistics in China, 2015. CA Cancer J Clin. 2016;66(2):115-132.

6. Fukunaga A, et al. CD8+ tumor-infiltrating lymphocytes together with CD4+ tumor-infiltrating lymphocytes and dendritic cells improve the prognosis of patients with pancreatic adenocarcinoma. Pancreas. 2004;28(1):e26-e31.

7. Sharma P, Wagner K, Wolchok JD, Allison JP. Novel cancer immunotherapy agents with survival benefit: recent successes and next steps. Nat Rev Cancer. 2011;11(11):805-812.

8. Vonderheide RH, Bayne LJ. Inflammatory networks and immune surveillance of pancreatic carcinoma. Curr Opin Immunol. 2013;25(2):200-205

9. Hiraoka N, Onozato K, Kosuge T, Hirohashi S. Prevalence of FOXP3+ regulatory T cells increases during the progression of pancreatic ductal adenocarcinoma and its premalignant lesions. Clin Cancer Res. 2006;12(18):5423-5434.

10. Ene-Obong A, et al. Activated pancreatic stellate cells sequester CD8+ T cells to reduce their infiltration of the juxtatumoral compartment of pancreatic ductal adenocarcinoma. Gastroenterology. 2013;145(5):1121-1132.

11. Ino Y, et al. Immune cell infiltration as an indicator of the immune microenvironment of pancreatic cancer. Br J Cancer. 2013;108(4):914-923. 
12. Hu H, Hang JJ, Han T, Zhuo M, Jiao F, Wang LW. The M2 phenotype of tumor-associated macrophages in the stroma confers a poor prognosis in pancreatic cancer. Tumour Biol. 2016;37(7):8657-8664

13. Blando J, et al. Comparison of immune infiltrates in melanoma and pancreatic cancer highlights VISTA as a potential target in pancreatic cancer. Proc Natl Acad Sci USA. 2019;116(5):1692-1697.

14. Wu X, Gao H, Ke W, Giese RW, Zhu Z. The homeobox transcription factor VentX controls human macrophage terminal differentiation and proinflammatory activation. J Clin Invest. 2011;121(7):2599-2613.

15. Zhu Y, et al. Tissue-resident macrophages in pancreatic ductal adenocarcinoma originate from embryonic hematopoiesis and promote tumor progression. Immunity. 2017;47(2):323-338.e6.

16. Mantovani A, Marchesi F, Malesci A, Laghi L, Allavena P. Tumour-associated macrophages as treatment targets in oncology. Nat Rev Clin Oncol. 2017;14(7):399-416.

17. Wu X, Gao H, Bleday R, Zhu Z. Homeobox transcription factor VentX regulates differentiation and maturation of human dendritic cells. J Biol Chem. 2014;289(21):14633-14643.

18. Le Y, Gao H, Bleday R, Zhu Z. The homeobox protein VentX reverts immune suppression in the tumor microenvironment. Nat Commun. 2018;9(1):2175.

19. Gordon S. Phagocytosis: an immunobiologic process. Immunity. 2016;44(3):463-475.

20. Bian Z, et al. Cd47-Sirp $\alpha$ interaction and IL-10 constrain inflammation-induced macrophage phagocytosis of healthy self-cells. Proc Natl Acad Sci USA. 2016;113(37):E5434-E5443.

21. Gordon SR, et al. PD-1 expression by tumour-associated macrophages inhibits phagocytosis and tumour immunity. Nature. 2017;545(7655):495-499.

22. Willingham SB, et al. The CD47-signal regulatory protein alpha (SIRPa) interaction is a therapeutic target for human solid tumors. Proc Natl Acad Sci USA. 2012;109(17):6662-6667.

23. Zhong YF, Holland PW. The dynamics of vertebrate homeobox gene evolution: gain and loss of genes in mouse and human lineages. BMC Evol Biol. 2011;11:169.

24. Mantovani A, Bottazzi B, Colotta F, Sozzani S, Ruco L. The origin and function of tumor-associated macrophages. Immunol Today. 1992;13(7):265-270.

25. Franklin RA, et al. The cellular and molecular origin of tumor-associated macrophages. Science. 2014;344(6186):921-925

26. Feng M, Jiang W, Kim BYS, Zhang CC, Fu YX, Weissman IL. Phagocytosis checkpoints as new targets for cancer immunotherapy. Nat Rev Cancer. 2019;19(10):568-586.

27. Leidi M, et al. M2 macrophages phagocytose rituximab-opsonized leukemic targets more efficiently than $\mathrm{m} 1$ cells in vitro. $J$ Immunol. 2009;182(7):4415-4422.

28. Kneidl J, et al. Soluble CD163 promotes recognition, phagocytosis and killing of Staphylococcus aureus via binding of specific fibronectin peptides. Cell Microbiol. 2012;14(6):914-936.

29. Moretti PA, Davidson AJ, Baker E, Lilley B, Zon LI, D'Andrea RJ. Molecular cloning of a human Vent-like homeobox gene. Genomics. 2001;76(1-3):21-29.

30. Gao H, Wu B, Giese R, Zhu Z. Xom interacts with and stimulates transcriptional activity of LEF1/TCFs: implications for ventral cell fate determination during vertebrate embryogenesis. Cell Res. 2007;17(4):345-356.

31. Gao H, Le Y, Wu X, Silberstein LE, Giese RW, Zhu Z. VentX, a novel lymphoid-enhancing factor/T-cell factor-associated transcription repressor, is a putative tumor suppressor. Cancer Res. 2010;70(1):202-211.

32. Mosser DM, Edwards JP. Exploring the full spectrum of macrophage activation. Nat Rev Immunol. 2008;8(12):958-969.

33. Bronte V, Murray PJ. Understanding local macrophage phenotypes in disease: modulating macrophage function to treat cancer. Nat Med. 2015;21(2):117-119.

34. Gao H, Wu X, Sun Y, Zhou S, Silberstein LE, Zhu Z. Suppression of homeobox transcription factor VentX promotes expansion of human hematopoietic stem/multipotent progenitor cells. J Biol Chem. 2012;287(35):29979-29987.

35. Kamada N, et al. Unique CD14 intestinal macrophages contribute to the pathogenesis of Crohn disease via IL-23/IFN-gamma axis. J Clin Invest. 2008;118(6):2269-2280.

36. Rogler G, et al. Isolation and phenotypic characterization of colonic macrophages. Clin Exp Immunol. 1998;112(2):205-215.

37. Eruslanov EB, et al. Tumor-associated neutrophils stimulate $\mathrm{T}$ cell responses in early-stage human lung cancer. J Clin Invest. 2014;124(12):5466-5480.

38. Mittal VK, Bhullar JS, Jayant K. Animal models of human colorectal cancer: Current status, uses and limitations. World J Gastroenterol. 2015;21(41):11854-11861.

39. Wong HH, Chu P. Immunohistochemical features of the gastrointestinal tract tumors. J Gastrointest Oncol. 2012;3(3):262-284. 